\title{
Tumores músculo esqueléticos, recomendaciones durante la pandemia de COVID-19
}

\author{
Musculoskeletal tumors, recommendations during the COVID-19 pandemic
}

\author{
Víctor Manuel Sepúlveda Oyervides*
}

\begin{abstract}
* Director del Comité de Tumores Músculo-Esqueléticos del Comité Científico FEMECOT. Médico de base en Traumatología y Ortopedia en el Hospital Regional del ISSSTE de Monterrey, Nuevo León, México. Profesor titular de los programas de residencia y de subespecialidad en cirugía de cadera y de rodilla en el Hospital Regional del ISSSTE de Monterrey, Nuevo León, México.
\end{abstract}

Correspondencia: Dr. Víctor Manuel Sepúlveda Oyervides. Centro Médico González.

Calle Doctor Guajardo No. 160, Despacho 203, Col. Los Doctores, 64710, Monterrey,

Nuevo León, México. Correo electrónico: drvictorsepulveda@hotmail.com

\section{RESUMEN}

El 31 de diciembre de 2019 se reportan los primeros casos en Wuhan, China de una nueva infección de vías respiratorias con gran transmisión y alta letalidad de origen desconocido, más tarde se identifica como productor un nuevo coronavirus clasificado como SARS-CoV-2 ahora conocido formalmente como COVID-19. Siendo estos tiempos históricos, esto ha representado todo un reto para el área médica mundial, ya que involucra a todas las especialidades médicas. En el presente artículo se hacen recomendaciones en relación al manejo de los tumores óseos benignos, localmente agresivos y malignos durante la pandemia de COVID-19.

Palabras clave: Tumores óseos, COVID-19, recomendaciones, ortopedia oncológica, cirugía tumoral.

\section{ABSTRACT}

December 31, 2019 marks the date when a new highly transmissible and highly lethal airway infection is reported in Wuhan, China. The pathogen responsible for this illness was then determined to be a novel coronavirus which was labelled SARSCoV-2 now known formally as COVID-19. These have proven to be historic times, which have posed a challenge to the global medical community including all specialties. The present article emits recommendations regarding the management of benign, locally aggressive, and malignant bone tumors during the COVID-19 pandemic.

Keywords: Bone tumors, COVID-19, recomendations, oncology orthopedics, tumoral surgery.

En el contexto de la atención de una lesión tumoral ósea que se presenta como caso clínico en consulta o en el área de urgencias durante la pandemia de COVID-19 debemos dividirla en varios escenarios:

El primero sería cuando detectamos una lesión benigna de tipo S1 o S2 de la clasificación de Enneking ${ }^{1}$ en la consulta regular; en estos casos 
debemos hacer los estudios pertinentes de laboratorio y de imagen requeridos $^{2}$ para tener mayor certeza ante el probable diagnóstico de la misma (radiografías simples, TAC, RMN, etcétera) y postergar por ocho a 12 semanas efectuar algún tipo de cirugía en caso de estar indicada ${ }^{3}$ ya sea toma de biopsia ${ }^{4}$ o bien resección de la lesión con o sin reconstrucción del área afectada, recordando que algunos de estos procedimientos requieren anestesia general, ${ }^{5,6}$ lo que nos aumenta el riesgo de contaminación cruzada con COVID-19.

El segundo escenario sería cuando nos enfrentemos a lesiones localmente agresivas o S3 de acuerdo a la clasificación de Enneking; ${ }^{1}$ al ser estos casos observados por primera vez en la consulta externa, debemos tomar todos los estudios requeridos tanto de laboratorio como de imagen, descartar enfermedad metastásica pulmonar ${ }^{2}$ si las corticales óseas están íntegras; deberá postergarse la toma de la biopsia por ocho semanas a que pase el pico máximo de la pandemia si la cortical está destruida; al observar masa de tejidos blandos debe programarse biopsia ${ }^{4}$ con aguja fina o trocar dependiendo de la experiencia del cirujano, ésta debe efectuarse de preferencia en forma ambulatoria para disminuir el riesgo de contaminación intrahospitalaria de COVID-19. Una vez teniendo el resultado anatomopatológico se debe valorar el costo beneficio de efectuar una cirugía más amplia o de reconstrucción del área afectada, debiendo realizar pruebas de COVID-19 en un lapso no mayor de tres días previos a efectuar el tratamiento definitivo. En algunos de estos casos una espera de al menos seis semanas puede ser pertinente sin poner en riesgo el pronóstico del paciente. ${ }^{7,8,9}$

El tercer escenario corresponde a cuando nos enfrentamos a las lesiones malignas. Al presentarse un caso en la consulta debemos hacer el trabajo de estudio con sus respectivos laboratorios y estudios de imagen, así como la exploración por TAC $^{2}$ o de los pulmones o de un PET-CT. Es posible efectuar de forma ambulatoria la toma de biopsia ${ }^{4}$ para tener un diagnóstico anatomopatológico, esto previo a la toma de PCR no mayor de tres días para valorar COVID-19 prebiopsia en el paciente. Como ejemplo, en estos pacientes en caso de resultar osteosarcoma en la biopsia y el paciente está sujeto a un protocolo de quimioterapia neoadyuvante previo al tratamiento quirúrgico definitivo, este paciente debe recibir dicho protocolo a pesar del riesgo de COVID-19, haciéndole saber al paciente y familiares del riesgo por la inmunosupresión de la quimioterapia como efecto secundario y que debe estar en un protocolo de aislamiento familiar más estrecho.

El cuarto escenario corresponde al de un paciente con sarcoma ya diagnosticado y que durante la pandemia completa sus ciclos de quimioterapia neoadyuvante. Este paciente debe ser operado de acuerdo al programa quirúrgico normal planeado, ya que esto tiene tiempos muy estrechos para el protocolo de manejo de los sarcomas que no pueden ser prolongados, en estos pacientes también se debe realizar prueba COVID-19 no más de tres días antes de la cirugía debiendo repetirse ésta al momento del alta hospitalaria. 
El quinto escenario es cuando un paciente abre su cuadro tumoral con una fractura patológica; los estudios pertinentes de laboratorio e imagen así como TAC de pulmón y prueba de PCR para COVID-19 deben hacerse desde el punto de vista del tumor; si se trata de fractura por tumor benigno, dependiendo del caso, se puede manejar en forma conservadora, ya sea inicialmente o bien en forma definitiva; si se determina tratamiento quirúrgico estando en tiempo agudo la pandemia la recomendación de hacerlo es alrededor de ocho semanas después de la fractura siempre y cuando no se comprometa al paciente de acuerdo al sitio primario de la lesión. Una forma temporal y rápida de manejar a estos pacientes y que puede ser ambulatoria es la colocación de un fijador externo temporal para estabilidad de la fractura y posteriormente hacer el tratamiento definitivo. En el caso de que la lesión se trate de un tumor maligno que abre con una fractura patológica siendo esto ya de por sí una situación muy seria y compleja en tiempo de la pandemia de COVID-19, se deben practicar como en todos los casos estudios de laboratorio e imagen aunados a una valoración de extensión tumoral y de enfermedad metastásica por medio de PET-CT. ${ }^{2} \mathrm{Si}$ encontramos extensión tumoral dispersa, se debe proceder a una biopsia transoperatoria ${ }^{4} \mathrm{y}$, dependiendo del resultado, seguir con una cirugía radical tipo amputación o desarticulación mayor, ya que se trata de una verdadera emergencia quirúrgica. Si lo permite el resultado anatomopatológico y cabe la posibilidad de aplicar algún tipo de quimioterapia neoadyuvante o radioterapia en su caso, se puede colocar en forma temporal fijadores externos para dar estabilidad a la fractura corriendo el riesgo de contaminación por COVID-19, agregada primero por la inmunosupresión del paciente por el tumor maligno y segundo por el tratamiento, ya sea el caso de quimioterapia neoadyuvante y/o radioterapia. Hay artículos ${ }^{10}$ que reportan que en centros hospitalarios europeos bajaron los procedimientos quirúrgicos electivos para sarcomas óseos hasta $92 \%$ durante la pandemia por COVID-19.

\section{BIBLIOGRAFÍA}

1. Enneking WF, Spanier SS, Goodman MA. A system for the surgical staging of musculosqueletal sarcoma. Clin Orthop Relat Res. 2003; (415): 4-18.

2. Rougraff BT. Imaging of musculoskeletal lesions. Musculoskeletal Tumors. OKU AAOS, 2007.

3. Chang Liang Z, Wang W, Murphy D, Po Hu JH. Novel coronavirus and orthopaedic surgey. J Bone Jt Surg. 2020; 1: 1 Ap. 7.

4. Aboulafia AJ, Levine AM, Scmidt DP. Musculoskeletal tumors. OKU. AAOS 2007.

5. Caputo KM, Byrick R, Chapman MG, Orser BJ, Orser BA. Intubation of SARS patients: infection and perspectives of healthcare workers. Can J Anesth. 2006; 53 (2): 122-129.

6. Joo KH, Song Wook KJ, Kim TY. Scientific Committee of the Korean Society of Anesthesiologists. Recommendations for anesthesia in patients suspected of Covid-19 coronavirus infection. Korean $\mathrm{J}$ Anesthesiol. 2020; 73 (2): 89-91.

7. Coccolini F, Perrone G, Chiarugi M, Di Marzo F, Ansaloni L, Scandroglio I, et al. Surgery in Covid-19 patients: operational directives. World J Emerg Surg. 2020; 15: 25.

8. Guo X, Wang J, Hu D, Wu L, Gu L, Wang Y, et al. Survey of Covid-19 disease among orthopaedic surgeons in Wuhan. People's Republic of China. J. Bone and Joint Surg Am. 2020; 102 (10): 847-854.

9. Brat GA, Hersey SP, Chabra K, Gupta A, Scott J. Protecting surgical teams during the Covid-19 outbreak: a narrative review and clinical considerations. Ann Surg. 2020 (ePub ahead of print). 
10. Liebensteiner MC, Khosrani I. Hirschmann MT, Heuberer PR, The board of the AGA- Society of Arthroscopy and Joint Surgery Thaler M. Massive cutback in orthopaedic healthcare services due to the COVID-19 pandemic. Knee Surgery Sports Traumatology, Arthroscopy. 2020. (https://doi. org/10.1007/500167-020-06032-2) 30 April 2020. 